Pacific Journal of Mathematics

NILPOTENCY CLASS OF A MAP AND STASHEFF'S 


\title{
NILPOTENCY CLASS OF A MAP AND STASHEFF'S CRITERION
}

\author{
C. S. HoO
}

Let $f: X \rightarrow Y$ be a map and let $e: \Sigma \Omega X \rightarrow X$ be the map whose adjoint is $1_{2 x}$. Then we prove the following results.

Theorem 1. nil $f \leqq 1$ if and only if $f e r: \Sigma \Omega X \vee \Sigma \Omega X \rightarrow Y$ can be extended to $\Sigma \Omega X \times \Sigma \Omega X$.

Theorem 2. Let $X$ be an $H^{\prime}$-space. Then nil $f \leqq 1$ if and only if $f \nabla: X \vee X \rightarrow Y$ can be extended to $X \times X$.

Theorem 3. nil $f=\operatorname{nil}(f e)$.

Theorem 1 may be regarded as an extension of Stasheff's criterion for a loop space to be homotopy-commutative. These theorems may all be regarded as extensions of Stasheff's criterion in various ways. We also discuss the duals of these results. Theorem 3 dualises, but the others do not. A sample result in the dual situation is

Theorem. conil $f \leqq \Sigma w$ cat $\left(e^{\prime} f\right)$ where $e^{\prime}: Y \rightarrow \Omega \Sigma Y$ is the adjoint of $1_{\Sigma Y}$.

In this paper we shall work in the category $\mathscr{T}$ of spaces with base point and having the homotopy type of countable $C W$ complexes. All maps and homotopies shall respect base points. The maps of our category $\mathscr{T}$ shall be homotopy classes of maps, but for simplicity we shall use the same symbol for a map and its homotopy class. Given spaces $X, Y$, we denote the set of homotopy classes of maps from $X$ to $Y$ by $[X, Y]$. We have an isomorphism $\tau:[\Sigma X, Y] \rightarrow[X, \Omega Y]$ where $\Sigma, \Omega$ are the suspension and loop functors respectively. We denote $\tau\left(1_{\Sigma X}\right)$ by $e^{\prime}$ and $\tau^{-1}\left(1_{\Omega X}\right)$ by $e$.

1. For convenience let us recall some notions of Peterson's theory of structures [7]. We shall follow the definitions and notations of [4]. Let $\mathscr{C}$ be a category. By a left structure system $\mathscr{L}$ over $\mathscr{C}$ we mean $\mathscr{L}=(L, W, S ; d, j)$ where $L, W, S: \mathscr{C} \rightarrow \mathscr{S}$ are covariant functors and $d: W \rightarrow L, j: W \rightarrow S$ are natural transformations. Given an object $X$ of $\mathscr{C}$ we say that $X$ is $\mathscr{P}$-structured if there exists a map $\varphi: S X \rightarrow L X$ such that $\varphi j(X) \simeq d(X)$. Given a category $\mathscr{C}$, we have a category $\mathscr{C}^{2}$ of pairs. An object of $\mathscr{C}^{2}$ is a map $f: X \rightarrow Y$ of $\mathscr{C}$, and given objects $f: X_{1} \rightarrow X_{2}, g: Y_{1} \rightarrow Y_{2}$ of $\mathscr{C}^{2}$, a map $(u, v)$ : $f \rightarrow g$ is a pair of maps $u: X_{1} \rightarrow Y_{1}, v: X_{2} \rightarrow Y_{2}$ such that $g u=v f$. We have convariant functors $D_{0}, D_{1}: \mathscr{C}^{2} \rightarrow \mathscr{C}$ given by $D_{0}(f)=Y, D_{1}(f)=$ 
$X$ where $f: X \rightarrow Y$. Also given $(u, v): f \rightarrow g$, we have $D_{0}(u, v)=v$, $D_{1}(u, v)=u$. We have a natural trasformation $G: D_{1} \rightarrow D_{0}$ given by $G(f)=f$ for $f \in \mathscr{C}^{2}$. Given a left structure $\mathscr{P}=(L, W, S ; d, j)$ over $\mathscr{C}$, we have a left structure $\mathscr{C}^{2}=\left(L D_{0}, W D_{1}, S D_{1} ;\left(d D_{0}\right)(W G), j D_{1}\right)$ over $\sigma^{2}$. Given an object $f$ of $\mathscr{C}^{2}$, we shall say that $f$ is $\mathscr{L}$-structured if it is ${ }^{2}$-structured. It is easily seen that if $f: X \rightarrow Y$ is an object of $\mathscr{C}^{2}$, and $X$ or $Y$ is $Z$-structured, then $f$ is $\mathscr{C}$-structured.

We have the left structure $H=\left(1, \bigvee_{i=1}^{2}, \prod_{i=1}^{2}, \nabla, j\right)$ over $\mathcal{S}$, where 1 is the identity functor of $\mathscr{S}, V_{i=1}^{2}$ is the wedge product, $\Pi_{i=1}^{2}$ is the cartesian product and $\nabla, j$ are the folding and inclusion natural transformations respectively. We observe that a space $X$ is $H$-structured precisely if it is an $H$-space. Also a map $f: X \rightarrow Y$ is $H$-structured if and only if $f \nabla: X \vee X \rightarrow Y$ extends to $X \times X$.

2. Let $\mathscr{C}=(L, W, S ; d, j)$ be a left structure system over a category $\mathscr{C}$. Let $f: X \rightarrow Y, g: Y \rightarrow Z$ be maps. Then it is easily seen that if $f$ is $Z$-structured or $g$ is $f$-structured, then $g f$ is structured.

We recall that in [1], there is defined a generalized Whitehead product [,]: $[\Sigma A, X] \times[\Sigma B, X] \rightarrow[\Sigma(A \wedge B), X]$ where $A, B, X$ are spaces and $A \wedge B$ is the smashed product. Now suppose $X$ is an $H$ space. Then we have a generalized Samelson product (see [2]) $\langle$, $\rangle$ : $[A, X] \times[B, X] \rightarrow[A \wedge B, X]$. These homotopy operations are related in the following way. Suppose $\alpha$ is an element of $[\Sigma A, X], \beta$ is an element of $[\Sigma B, X]$ where $A, B, X$ are spaces. Then

$$
\tau[\alpha, \beta]=\langle\tau(\alpha), \tau(\beta)\rangle .
$$

We shall also make the following convention. Let $f: X \rightarrow Y$ be a map. Then we have an $H$-map $\Omega f: \Omega X \rightarrow \Omega Y$. We shall write nil $f$ for nil $\Omega f$ (see [3] for definitions). Similarly, we have an $H^{\prime}$-map $\Sigma f: \Sigma X \rightarrow \Sigma Y$. We shall write conil $f$ for conil $\Sigma f$.

Theorem 1. Let $f: X \rightarrow Y$ be a map. Then nil $f \leqq 1$ if and only if $f e^{17}: \Sigma \Omega X \vee \Sigma \Omega X \rightarrow Y$ can be extended to $\Sigma \Omega X \times \Sigma \Omega X$.

Proof. Let $c: \Omega X \times \Omega X \rightarrow \Omega X$ be the basic commutator of $\Omega X$. Then nil $f \leqq 1$ if and only if $(\Omega f) c \simeq *$. Let $i_{1}, i_{2}: \Sigma \Omega X \rightarrow \Sigma \Omega X \vee \Sigma \Omega X$ be the inclusions in the first and second coordinates respectively. Then we have a generalized Whitehead product

$$
\left[i_{1}, i_{2}\right] \in[\Sigma(\Omega X \wedge \Omega X), \Sigma \Omega X \vee \Sigma \Omega X] .
$$

Now $\Sigma \Omega X \times \Sigma \Omega X$ is homotopically equivalent to 


$$
(\Sigma \Omega X \vee \Sigma \Omega X) \bigcup_{\left[i_{1}, i_{2}\right]} C \Sigma(\Omega X \times \Omega X)
$$

(see [1]), so that $f e \nabla$ extends to $\Sigma \Omega X \times \Sigma \Omega X$ if and only if $f e \nabla\left[i_{1}, i_{2}\right]=0$, that is, $[f e, f e]=0$. Now $\tau[f e, f e]=\langle\Omega f, \Omega f\rangle$ and

$$
q^{\sharp}\langle\Omega f, \Omega f\rangle=c(\Omega f \times \Omega f) \simeq(\Omega f) c
$$

where the first $c$ denotes the commutator $\Omega Y \times \Omega X \rightarrow \Omega Y$ and the second $c$ denotes the commutor $\Omega X \times \Omega X \rightarrow \Omega X$ and $q: \Omega Y \times \Omega Y \Omega Y \wedge \Omega Y$ is the projection. Since $\tau$ is an isomorphism and $q^{\sharp}$ is a monomorphism, it follows that $f e]^{7}$ extends to $\Sigma \Omega X \times \Sigma \Omega X$ if and only if nil $f \leqq 1$.

REMARK. If we take $f$ to be the identity map of $X$, then the theorem says that nil $X \leqq 1$ if and only if $e^{7}: \Sigma \Omega X \vee \Sigma \Omega X \rightarrow X$ extends to $\Sigma \Omega X \times \Sigma Q X$, which is just Stasheff's criterion for the homotopy-commutativity of a loop space (see [8]). We also observe that the statement that $f e^{\prime 7}$ extends to $\Sigma Q X \times \Sigma Q X$ is just the statement that $f e$ can be $H$-structured.

Theorem 2. Let $f: X \rightarrow Y$ be a map where $X$ is an $H^{\prime}$-space. Then nil $f \leqq 1$ if and only if $f \nabla: X \vee X \rightarrow Y$ can be extended to $X \times X$.

In view of the fact that $f \nabla$ can be extended if and only if $f$ can be $H$ structured, Theorem 2 will follow from Theorem 1 and the following lemma.

Lemma. Let $f: X \rightarrow Y$ be a map where $X$ is an $H^{\prime}$-space. Then $f$ is $H$-structured if and only if $f e: \Sigma \Omega X \rightarrow Y$ is $H$-structured.

Proof. We need only show that if $f e$ is $H$-structured then $f$ is $H$-structured. Suppose $f e$ can be $H$-structured. Then we can find a map $\varphi: \Sigma \Omega X \times \Sigma \Omega X \rightarrow Y$ such that $\varphi j \simeq \nabla(f e \vee f e)=f e \nabla$. Since $X$ is an $H^{\prime}$ :space we have a map $s: X \rightarrow \Sigma \Omega X$ such that $e s \simeq 1_{X}$. Then $\varphi(s \times s): X \times X \rightarrow Y$ is an $H$-structure for $f$. In fact $\varphi(s \times s) j=$ $\phi j(s \vee s) \simeq f e \nabla(s \vee s)=f e s \nabla \simeq f \nabla$.

REMARK. Theorems 1 and 2 imply that nil $e \leqq 1$ if and only if $\Omega X$ is homotopy-commutative, that is, if and only if nil $X \leqq 1$. In fact, we always have nil $X=$ nil $e$. This fact follows from the next result.

Theorem 3. Let $f: X \rightarrow Y$ be a map. Then nil $f=\operatorname{nil}(f e)$.

Proof. Since we always have nil $(f e) \leqq$ nil $f$, it suffices to show that $\operatorname{nil} f \leqq \operatorname{nil}(f e)$. Suppose $\operatorname{nil}(f e) \leqq n$. Then $(\Omega f)(\Omega e) c_{n+1} \simeq *$ 
where $c_{n+1}:(\Omega \Sigma \Omega X)^{n+1} \rightarrow \Omega \Sigma \Omega X$ is the commutator map of weight $(n+1)$. Then we have

$$
(\Omega f) c_{n+1}(\Omega e \times \cdots \times \Omega e) \simeq *
$$

where $c_{n+1}:(\Omega X)^{n+1} \rightarrow \Omega X$ is also the commutator map of weight $(n+1)$. Consider the map $e^{\prime}: \Omega X \rightarrow \Omega \Sigma \Omega X$ such that $e^{\prime}=\tau\left(1_{\Omega \Sigma X}\right)$. Clearly $(\Omega e) e^{\prime}=1_{\Omega \Sigma}$. Hence we have $(\Omega f) c_{n+1} \simeq *$, that is, nil $f \leqq n$. This proves the theorem.

3. We now consider the dual situation. It is clear that Theorem 3 dualises immediately to give the following result.

THEOREM 4. Let $f: X \rightarrow Y$ be a map and let $e^{\prime}: Y \rightarrow \Omega \Sigma Y$ be the adjoint of $1_{\Sigma X}$. Then conil $f=\operatorname{conil}\left(e^{\prime} f\right)$.

Let us first define a right structure system over a category $\mathscr{C}$. By this we shall mean $\mathscr{R}=(R, P, T ; d, j)$ where $R, P, T: \mathscr{C} \rightarrow \mathscr{T}$ are covariant functors and $d: R \rightarrow P, j: T \rightarrow P$ are natural transformations. Given an object $X \in \mathscr{C}$, we say that $X$ is $\mathscr{R}$-structured if there exists a map $\varphi: R X \rightarrow T X$ such that $j(X) \varphi \simeq d(X)$. Given a right structure $\mathscr{R}=(R, P, T ; d, j)$ over $\mathscr{C}$, we can form a right structure $\mathscr{R}^{2}=\left(R D_{1}, P D_{0}, T D_{0} ;\left(d D_{0}\right)(R G), j D_{0}\right)$ over $\mathscr{C}^{2}$. We shall say that an element $f: X \rightarrow Y$ of $\mathscr{C}^{2}$ is $\mathscr{R}$-structured if it is $\mathscr{R}^{2}$ structured. It is easily checked that if $X$ or $Y$ is $\mathscr{R}$-structured, then $f$ is $\mathscr{R}$-structured.

The dual of the $H$-structure is the $H^{\prime}$-structure $\left(1, \prod_{i=1}^{2}, \mathrm{~V}_{i=1}^{2} ; \Delta, j\right)$, a right structure over $\mathscr{T}$. Clearly a space $X$ is $H^{\prime}$-structured if and only if it is an $H^{\prime}$-space. Also a map $f: X \rightarrow Y$ is $H^{\prime}$-structured if and only if $\Delta f: X \rightarrow Y^{2}$ can be compressed into $Y \vee Y$. The dual of Theorem 1 would read: conil $f \leqq 1$ if and only if $\Delta e^{\prime} f: X \rightarrow(\Omega \Sigma Y)^{2}$ can be compressed into $\Omega \Sigma Y \vee \Omega \Sigma Y$. This, however, is false (see [5]). But in this case, we can generalize the $H^{\prime}$-structure to another familiar right structure, namely the $n$-cat structure $\left(1, \prod_{i=1}^{n+1} ; T_{1}, \Delta, j\right)$ over $\mathscr{T}$, where $T_{1}$ is the fat wedge functor. Thus the 1-cat structure is precisely the $H^{\prime}$-structure. Given a space $X$, we have cat $X \leqq n$ if there exists a map $\varphi: X \rightarrow T_{1}(X, \cdots, X)$ such that $j \varphi \simeq \Delta: X \rightarrow X^{n+1}$. Given a map $f: X \rightarrow Y$, we have cat $f \leqq n$ if $\Delta f: X \rightarrow Y^{n+1}$ can be compressed into $T_{1}(Y, \cdots, Y)$.

Given a right structure system $\mathscr{R}=(R, P, T ; d, j)$ over $\mathscr{C}$, let us consider the cofibre of $j: T \rightarrow P$. Suppose the cofibre of $j$ is $q: P \rightarrow Q$. Let $j_{w} \rightarrow P$ be the fibre of $q$. Then we obtain a right structure system $\mathscr{R}_{w}=\left(R, P, T_{w} ; d, j_{w}\right)$ over $\mathscr{C}$, called the associated weak structure. We shall say that an object $X \in \mathscr{C}$ is weakly $\mathscr{R}$ - 
structured if it can be $\mathscr{R}_{w}$-structured. Clearly, given a map $f: X \rightarrow Y$ we have $w$ cat $f \leqq n$ if $q \Delta f \simeq *$ where $q: Y^{n+1} \rightarrow \bigwedge_{i=1}^{n+1} Y$ is the projection onto the smashed product. Given a right structure $\mathscr{R}=(R$, $P, T ; d, j)$ over $\mathscr{C}$, we have a right structure $\Sigma \mathscr{R}=(\Sigma R, \Sigma P, \Sigma T ; \Sigma d$, $\Sigma j$ ) over $\mathscr{C}$, where $\Sigma$ is the suspension functor. Clearly, if $f$ is $\mathscr{R}$ structured, it is $\Sigma \mathscr{R}$-structured and it is weakly $\mathscr{R}$-structured. Thus $\Sigma w$ cat $f \leqq w$ cat $f \leqq$ cat $f$ for any map $f$.

Let $f: X \rightarrow Y, g: Y \rightarrow Z$ be maps. Then it is easily seen that cat $(g f) \leqq \min \{$ cat $f$, cat $g\}$ and $w$ cat $(g f) \leqq \min \{w$ cat $f, w$ cat $g\}$.

THeOREM 5. Let $f: X \rightarrow Y$ be a map and let $e^{\prime}: Y \rightarrow \Omega \Sigma Y$ be the adjoint of $1_{\Sigma Y}$. Then conil $f \leqq \Sigma w$ cat $\left(e^{\prime} f\right)$.

Proof. Suppose $\Sigma w$ cat $\left(e^{\prime} f\right) \leqq n$. Then $\Sigma\left(q \Delta e^{\prime} f\right) \simeq *$ where $q:(\Omega \Sigma Y)^{n+1} \rightarrow \bigwedge_{i=1}^{n+1} \Omega \Sigma Y$ is the projection. Let $c: \Sigma Y \rightarrow \bigvee_{i=1}^{n+1} \Sigma Y$ be the commutator map of weight $(n+1)$ for $\Sigma Y$. Then we can form a map $\bar{c}: Y^{n+1} \rightarrow \Omega\left(\bigvee_{i=1}^{n+1} \Sigma Y\right)$ such that $\bar{c}_{\Delta}=\tau(c)$ (see [5]). Since $\Sigma\left(q \Delta e^{\prime} f\right) \simeq *$, applying $\tau$ we have $\Omega \Sigma(q \Delta) e^{\prime} f \simeq *$. Consider the following diagram where each square is homotopy-commutative.

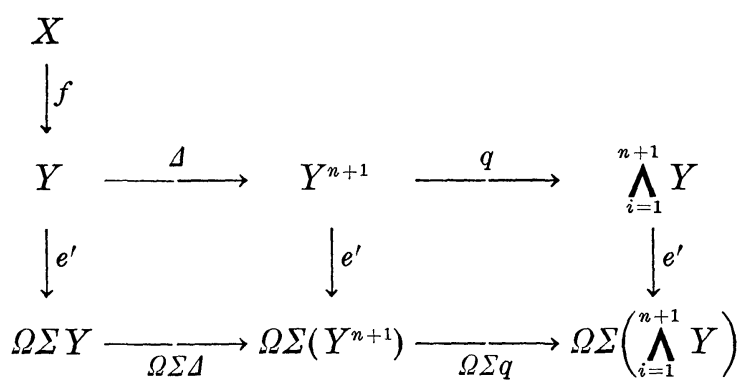

We have then that $e^{\prime} q \Delta f \simeq *$. Using Lemmas $4.1_{k}$ and $4.2_{k}$ of [5], it follows that $\bar{c}_{\Delta f} \simeq *$, that is, $\tau(c) f \simeq *$. Hence $c(\Sigma f) \simeq *$, and hence conil $f \leqq n$. This proves that conil $f \leqq \Sigma w$ cat $\left(e^{\prime} f\right)$.

Theorem 6. Let $f: X \rightarrow Y$ be a map where $Y$ is an $H$-space. Then cat $f=\operatorname{cat}\left(e^{\prime} f\right), w$ cat $f=w$ cat $\left(e^{\prime} f\right)$ where $e^{\prime}: Y \rightarrow \Omega \Sigma Y$ is the adjoint of $1_{\Sigma Y}$.

Proof. We need only show that cat $f \leqq \operatorname{cat}\left(e^{\prime} f\right)$, and

$$
w \text { cat } f \leqq w \text { cat }\left(e^{\prime} f\right) \text {. }
$$

Since $Y$ is an $H$-space, we have a map $r: \Omega \Sigma Y \rightarrow Y$ such that $r e^{\prime} \simeq 1_{Y}$. Then cat $f=\operatorname{cat}\left(r e^{\prime} f\right) \leqq \operatorname{cat}\left(e^{\prime} f\right)$ and $w$ cat $f=w$ cat $\left(r e^{\prime} f\right) \leqq w$ cat $\left(e^{\prime} f\right)$. 


\section{REFERENCES}

1. M. Arkowitz, The generalized Whitehead product, Pacific J. Math. 12 (1962), 7-23. 2. - Homotopy products for H-spaces, Michigan Math. J. 10 (1963), 1-9.

3. I. Berstein and T. Ganea, Homotopical nilpotency, Illinois J. Math. 5 (1961), 99-130. 4. I. Berstein and P. J. Hilton, Homomorphisms of homotopy structures, Topologie et geometric differentielle, Séminaire Ehresmann, April, 1963.

5. T. Ganea, P. J. Hilton and F. P. Peterson, On the homotopy-commutativity of loop spaces and suspensions, Topology 1 (1962), 133-141.

6. C. S. Hoo, A note on a theorem of Ganea, Hilton and Peterson, Proc. Amer. Math. Soc. 19 (1968), 909-911.

7. F. P. Peterson, Numerical invariants of homotopy type, Colloquium on algebraic topology, Aarhus Universitet, 1962, 79-83.

8. J. Stasheff, On homotopy abelian H-spaces, Proc. Camb. Phil. Soc. 57 (1961), 734745 .

Received January 23, 1968. This research was supported by NRC Grant A-3026.

UNIVERSITY OF ALBERTA

Edmonton, Alberta, Canada 


\section{PACIFIC JOURNAL OF MATHEMATICS}

\section{EDITORS}

H. ROYDEN

Stanford University

Stanford, California

\section{R. $R$ PHELPS}

University of Washington

Seattle, Washington 98105

\section{J. DugunduI}

Department of Mathematics

University of Southern California

Los Angeles, California 90007

RICHARD ARENS

University of California

Los Angeles, California 90024

\section{ASSOCIATE EDITORS}

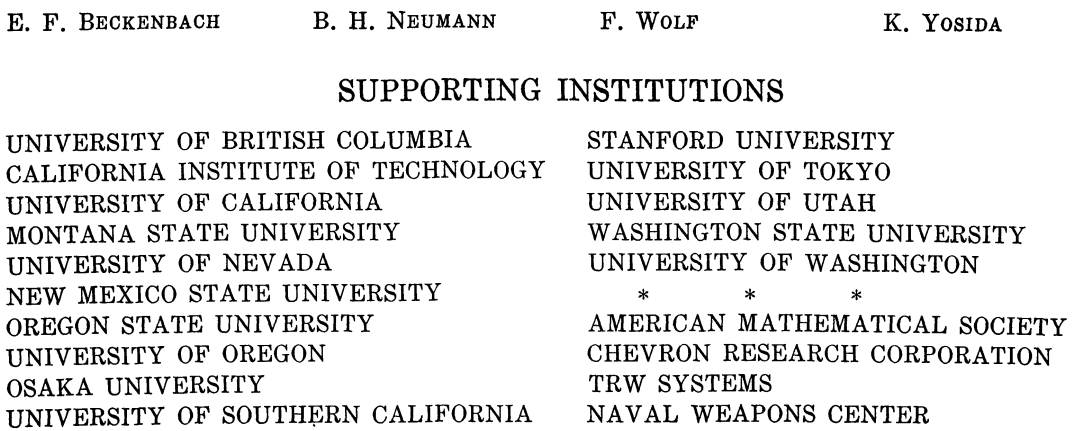

The Supporting Institutions listed above contribute to the cost of publication of this Journal, but they are not owners or publishers and have no responsibility for its content or policies.

Mathematical papers intended for publication in the Pacific Journal of Mathematics should be in typed form or offset-reproduced, double spaced with large margins. Underline Greek letters in red, German in green, and script in blue. The first paragraph or two must be capable of being used separately as a synopsis of the entire paper. It should not contain references to the bibliography. Manuscripts, in duplicate if possible, may be sent to any one of the four editors. Please classify according to the scheme of Math. Rev. 36, 1539-1546. All other communications to the editors should be addressed to the managing editor, Richard Arens, University of California, Los Angeles, California, 90024.

50 reprints are provided free for each article; additional copies may be obtained at cost in multiples of 50 .

The Pacific Journal of Mathematics is published monthly. Effective with Volume 16 the price per volume (3 numbers) is $\$ 8.00$; single issues, $\$ 3.00$. Special price for current issues to individual faculty members of supporting institutions and to individual members of the American Mathematical Society: $\$ 4.00$ per volume; single issues $\$ 1.50$. Back numbers are available.

Subscriptions, orders for back numbers, and changes of address should be sent to Pacific Journal of Mathematics, 103 Highland Boulevard, Berkeley, California, 94708.

PUBLISHED BY PACIFIC JOURNAL OF MATHEMATICS, A NON-PROFIT CORPORATION

Printed at Kokusai Bunken Insatsusha (International Academic Printing Co., Ltd.), 7-17, Fujimi 2-chome, Chiyoda-ku, Tokyo, Japan. 


\section{Pacific Journal of Mathematics}

Vol. 28, No. $2 \quad$ April, 1969

Richard Arens and Donald George Babbitt, The geometry of relativistic

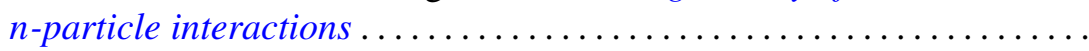

Kirby Alan Baker, Hypotopological spaces and their embeddings in lattices

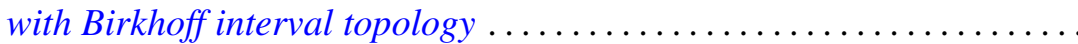

J. Lennart (John) Berggren, Finite groups in which every element is conjugate to its inverse ........................... 289

Beverly L. Brechner, Homeomorphism groups of dendrons . . . . . . . . . . . 295

Robert Ray Colby and Edgar Andrews Rutter, QF - 3 rings with zero singular ideal ................................. 303

Stephen Daniel Comer, Classes without the amalgamation property....... 309

Stephen D. Fisher, Bounded approximation by rational functions ......... 319

Robert Gaines, Continuous dependence for two-point boundary value problems..................................... 327

Bernard Russel Gelbaum, Banach algebra bundles ............... 337

Moses Glasner and Richard Emanuel Katz, Function-theoretic degeneracy criteria for Riemannian manifolds ...................... 351

Fletcher Gross, Fixed-point-free operator groups of order $8 \ldots \ldots \ldots \ldots 357$

Sav Roman Harasymiv, On approximation by dilations of distributions . . . . 363

Cheong Seng Hoo, Nilpotency class of a map and Stasheff's criterion ... . . 375

Richard Emanuel Katz, A note on extremal length and modutus.......... 381

H. L. Krall and I. M. Sheffer, Difference equations for some orthogonal polynomials ................................

Yu-Lee Lee, On the construction of lower radical properties ........... 393

Robert Phillips, Liouville's theorem........................... 397

Yum-Tong Siu, Analytic sheaf cohomology groups of dimension $n$ of

n-dimensional noncompact complex manifolds ..... . .

Michael Samuel Skaff, Vector valued Orlicz spaces. II...

James DeWitt Stein, Homomorphisms of $B^{*}$-algebras .... . .

Mark Lawrence Teply, Torsionfree injective modules .... . . .

Richard R. Tucker, The $\delta^{2}$-process and related topics. II .

David William Walkup and Roger Jean-Baptiste Robert Wets, Lifting

projections of convex polyhedra...

Thomas Paul Whaley, Large sublattices of a lattice. 